\title{
Усиление терагерцового излучения высокодобротными резонансными плазмонами в двухслойной периодической структуре на основе графена в режиме антикроссинга плазмонных мод
}

\author{
(C) О.В. Полищук ${ }^{1}$, Д.В. Фатеев ${ }^{1,2}$, В.В. Попов ${ }^{1}$ \\ ${ }^{1}$ Саратовский фрилиал Института радиотехники и электроники им. В.А. Котельникова Российской академии наук, \\ 410019 Саратов, Россия \\ ${ }^{2}$ Саратовский государственный университет, \\ 410012 Саратов, Россия \\ E-mail: polischuk.sfire@mail.ru, popov_slava@yahoo.co.uk
}

Поступила в Редакцию 24 апреля 2019 г.

В окончательной редакции 29 апреля 2019 г.

Принята к публикации 29 апреля 2019 г.

\begin{abstract}
Теоретически исследован спектр усиления терагерцового излучения в двухслойном структурированном активном графене, представляющем собой два одинаковых периодических массива графеновых микрополосок, разделенных тонким диэлектрическим барьерным слоем. Рассматриваемая система поддерживает оптические и акустические плазмонные моды. Резонансные частоты оптической и акустической мод меняются противоположным образом с изменением толщины изолирующего диэлектрического слоя, что делает возможным режим антикроссинга плазмонных мод. Показано, что исследуемая графеновая структура характеризуется сильным плазмонным откликом и гигантским усилением терагерцового излучения на частотах плазменного резонанса в окрестности режима антикроссинга между оптическими и акустическими плазмонными модами при комнатной температуре.
\end{abstract}

Ключевые слова: терагерцовое излучение, плазмон, графен, антикроссинг плазмонных мод.

DOI: 10.21883/FTP.2019.09.48131.14

\section{1. Введение}

Графен, двумерный монослой атомов углерода, является природным материалом с нулевой запрещенной зоной. В дополнение к его уникальным оптическим [1] и электронным свойствам, обусловленным бесщелевым линейным энергетическим спектром носителей заряда $[2,3]$, графен обладает сильным плазмонным откликом на терагерцовых (ТГц) частотах, что обусловлено как высокой плотностью, так и малой коллективной динамической массой свободных носителей заряда $[4,5]$. Использование плазменных колебаний носителей заряда в графене представляется привлекательным, поскольку позволяет сконцентрировать электромагнитное поле вблизи графена и таким образом значительно повысить эффективность взаимодействия ТГц излучения с графеном. Это является важным как с точки зрения изучения физических свойств самого графена, так и для создания ТГц устройств на основе графена.

В ряде работ была показана возможность усиления ТГц излучения оптически накачанным графеном [6-10] вследствие создания инвертированного распределения носителей заряда (электронов и дырок) в графене. После генерации электронно-дырочных пар в графене происходит их быстрая термализация с участием оптических фононов $[11,12]$. Ниже энергии оптического фонона существенным механизмом релаксации носителей является оже-рекомбинация [13-15]. Время жизни инвертированных носителей, измеренное в недавних эксперимен- тальных работах [16-19], находится в субпикосекундном диапазоне (130-250фс) при комнатной температуре и достигает сотен пикосекунд при низких температуpax [20]. В результате инверсии носителей заряда возникает отрицательная высокочастотная дифференциальная проводимость графена при достижении некоторого порогового значения оптической накачки [21]. Наличие отрицательной дифференциальной проводимости может приводить к стимулированной генерации ТГц плазмонов в графене [11]. Вынужденное излучение фотонов в инвертированном графене наблюдалось в ТГц диапазоне [12]. По сравнению с вынужденным излучением электромагнитных мод (фотонов), вынужденное излучение плазмонов при межзонных переходах носителей заряда в инвертированном графене является гораздо более сильным эффектом за счет малой групповой скорости плазмонов в графене и сильной локализации плазмонного поля вблизи графенового слоя [21].

Двухслойная графеновая структура представляет собой два параллельных графеновых слоя, разделенных тонким диэлектрическим барьером. Электромагнитные поля распространяющихся в этих слоях плазмонов взаимодействуют друг с другом, что приводит к образованию единой плазмонной моды в двухслойной графеновой структуре [22].

В данной статье рассматривается усиление ТГц волны посредством стимулированной генерации резонансных плазмонов в двухслойной периодической структуре на основе инвертированного графена. 


\section{2. Постановка задачи и метод решения}

Рассмотрим два параллельных бесконечных листа периодически структурированного графена, расположенных в плоскостях $y=0$ и $y=d$ и отделенных друг от друга слоем диэлектрика с вещественной диэлектрической проницаемостью $\varepsilon_{b}$ (рис. 1). Каждый лист структурированного графена представляет собой последовательность графеновых микрополосок шириной $w$ с периодом $L$. Вся структура расположена на поверхности подложки с вещественной диэлектрической ппроницаемостью $\varepsilon_{s}$. Окружающая среда при $y>d$ имеет диэлектрическую проницаемость $\varepsilon_{a}=1$ (вакуум). Каждая проводящая полоска периодического массива бесконечна в $z$-направлении.

Если характерные времена электрон-электронных и электрон-дырочных столкновений в графене малы по сравнению с временем рекомбинации носителей заряда, электроны и дырки можно описать равновесной функцией Ферми с квазиуровнями Ферми соответственно $E_{\mathrm{F}}$ и $-E_{\mathrm{F}}$ для электронов и дырок и эффективной температурой носителей заряда $T$. При достаточно сильном рассеянии носителей заряда на оптических фононах разогрев электронно-дырочной системы мал и можно полагать, что эффективная температура носителей заряда $T$ равна температуре кристаллической решетки графена $T_{0}$, $T=T_{0}$. В этом случае проводимость двумерных полосок инвертированного графена описывается комплексной динамической поверхностной проводимостью $[23,21]$

$$
\begin{aligned}
\sigma_{\mathrm{Gr}}(\omega)= & \sigma_{0} \frac{8 k_{\mathrm{B}} T_{0} \tau}{\pi \hbar(1-i \omega \tau)} \ln \left[1+\exp \left(\frac{E_{\mathrm{F}}}{k_{\mathrm{B}} T_{0}}\right)\right] \\
& +\sigma_{0}\left[\tanh \left(\frac{\hbar \omega-2 E_{\mathrm{F}}}{4 k_{\mathrm{B}} T_{0}}\right)\right. \\
& \left.-\frac{4 \hbar \omega}{i \pi} \int_{0}^{\infty} \frac{G\left(E, E_{\mathrm{F}}\right)-G\left(\hbar \omega / 2, E_{\mathrm{F}}\right)}{(\hbar \omega)^{2}-4 E^{2}} d E\right] .
\end{aligned}
$$

Здесь $\omega-$ круговая частота падающей электромагнитной волны, $e-$ заряд электрона, $\hbar-$ приведенная постоянная Планка, $k_{\mathrm{B}}$ - постоянная Больцмана, $\tau$ феноменологическое время рассеяния носителей заряда в графене, $\sigma_{0}=e^{2} / 4 \hbar$,

$$
G\left(E, E^{\prime}\right)=\frac{\sinh \left(E / k_{\mathrm{B}} T_{0}\right)}{\cosh \left(E / k_{\mathrm{B}} T_{0}\right)+\cosh \left(E^{\prime} / k_{\mathrm{B}} T_{0}\right)} .
$$

Первое слагаемое в формуле (1) описывает внутризонное диссипативное (друдевское) поглощение в графене, определяемое феноменологическим временем рассеяния носителей заряда в графене $\tau$. Второе слагаемое описывает межзонные переходы носителей заряда в графене.

Действительная часть проводимости (1), ответственная за диссипацию энергии в графене и учитывающая оба механизма рассеяния (внутризонное диссипативное

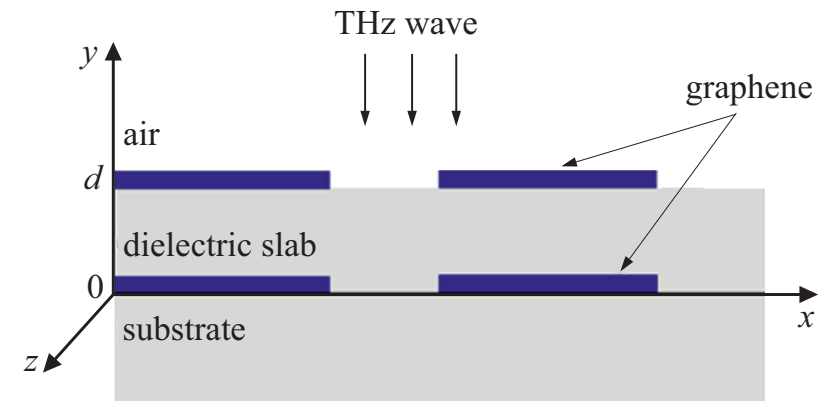

Рис. 1. Схематическое изображение двух периодов исследуемой структуры и система координат. Внешняя ТГц волна падает сверху в направлении нормали к плоскости структуры с поляризацией электрического поля поперек микрополосок графена (вдоль оси $x$ ).

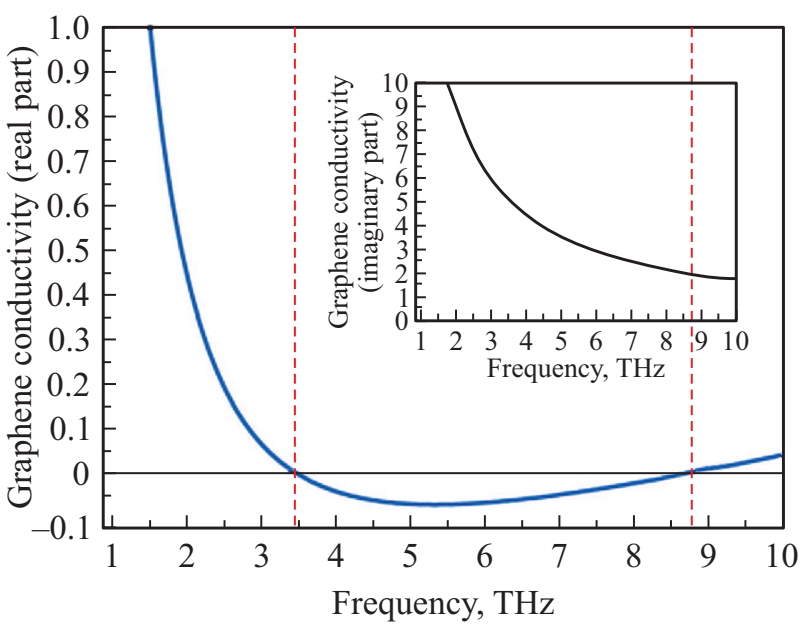

Рис. 2. Действительная часть проводимости инвертированного графена, нормированная на величину $\sigma_{0}=e^{2} / 4 h$, в зависимости от частоты падающей ТГц волны при заданной величине энергии квазиуровня Ферми 20 мэВ. Расчеты приведены для комнатной температуры и $\tau=1$ пс. На вставке - нормированная мнимая часть проводимости инвертированного графена.

рассеяние Друде и межзонное рассеяние вследствие генерации и рекомбинации электронно-дырочных пар), представлена на рис. 2. Действительная часть проводимости инвертированного графена (1) немонотонно зависит от частоты падающей электромагнитной волны и может принимать отрицательные значения даже при малой величине оптической накачки графена (соответствующей величине энергии квазиуровня Ферми 20 мэВ) и феноменологическом времени рассеяния носителей заряда в графене $\tau=1$ пс (соответствующем комнатной температуре) в широком терагерцовом диапазоне частот 3.5-8.75 ТГц, как показано вертикальными штриховыми линиями на рис. 2. В этом частотном диапазоне межзонные излучательные переходы носителей заряда в графене превалируют над суммарными потерями в графене. Вне этого частотного диапазона действительная часть проводимости инвертированного графена положительна 
(что соответствует режиму поглощения в графене), так как на меньших частотах доминируют внутризонные диссипативные потери, тогда как на частотах $>8.75$ ТГц, квант энергии $\hbar \omega$ превосходит по величине удвоенную энергию квазиуровня Ферми во втором слагаемом формулы (1). Мнимая часть проводимости графена (1), ответственная за резонансные частоты плазменных колебаний в графене, изменяется монотонно во всем ТГц диапазоне (см. вставку к рис. 2).

Будем считать, что плоская электромагнитная волна падает из вакуума нормально на плоскость структуры. Электрическое поле падающей волны $\mathbf{E}^{(0)} \exp \left[i k_{y}^{(0)} y-i \omega t\right]$, где $k_{y}^{(0)}=k_{0}=\omega / c, c-$ скорость света в вакууме, поляризовано поперек полосок решетки (вдоль оси $x$ ).

Задача решалась с использованием самосогласованного электродинамического подхода. В рассматриваемой геометрии индуцированное электрическое поле имеет ненулевые $x$ - и $y$-компоненты, тогда как индуцированное магнитное поле имеет только ненулевую $z$-компоненту (p-поляризация). В силу периодичности структуры в $x$-направлении, индуцированные магнитное и электрическое поля можно представить в виде ряда Фурье

$$
\begin{gathered}
H_{z}(x, y)=\sum_{m=-\infty}^{\infty} H_{z m}(y) \exp \left(i q_{m} x\right), \\
E_{x, y}(x, y)=\sum_{m=-\infty}^{\infty} E_{x m, y m}(y) \exp \left(i q_{m} x\right),
\end{gathered}
$$

где

$$
\begin{gathered}
H_{z m}(y)=\frac{1}{L} \int_{-L / 2}^{L / 2} H_{z}(x, y) \exp \left(-i q_{m} x\right), \\
E_{x m, y m}(y)=\frac{1}{L} \int_{-L / 2}^{L / 2} E_{x, y}(x, y) \exp \left(-i q_{m} x\right)
\end{gathered}
$$

- амплитуды $m$-й фурье-гармоники соответственно магнитного и электрического полей, $q_{m}=2 \pi m / L$ $(m= \pm 1, \pm 2, \pm 3 \ldots)$.

Используя уравнения (2) для каждой искомой компоненты поля, запишем систему уравнений Максвелла в каждой среде в фурье-представлении. В этом представлении граничные условия для тангенциальных составляющих электрического поля, $E_{x}$, и магнитного поля, $H_{z}$, записываются в виде

$$
\begin{gathered}
E_{x m}^{(b)}(0)=E_{x m}^{(s)}(0), \\
H_{z m}^{(b)}(0)-H_{z m}^{(s)}(0)=-I_{x m}(0)
\end{gathered}
$$

в плоскости $y=0$ и

$$
\begin{gathered}
E_{x m}^{(0)}(d)+E^{(0)} \exp \left(i k_{0} d\right)=E_{x m}^{(b)}(d), \\
H_{z m}^{(0)}(d)+H^{(0)} \exp \left(i k_{0} d\right)-H_{z m}^{(b)}(d)=I_{x m}(d)
\end{gathered}
$$

в плоскости $y=d$, где $I_{x m}(0)$ и $I_{x m}(d)-$ амплитуды $m$-й фурье-гармоники поверхностной плотности тока соответственно в двух плоскостях графеновых полосок. Индексы $a, b$ и $s$ относятся соответственно к окружающей среде (вакуум в рассматриваемом случае), барьерному слою и подложке. Амплитуды фурье-гармоник индуцированного магнитного поля в различных средах записываются в виде

$$
\begin{gathered}
H_{z m}^{(a)}=A_{m} \exp \left(i \alpha_{m}^{(a)} y\right), \quad y \geq d, \\
H_{z m}^{(b)}(y)=B_{m} \exp \left(i \alpha_{m}^{(b)} y\right)+C_{m} \exp \left(-i \alpha_{m}^{(b)} y\right), \quad 0 \leq y \leq d, \\
H_{z m}^{(s)}=D_{m} \exp \left(i \alpha_{m}^{(s)} y\right), \quad y \leq 0,
\end{gathered}
$$

где $\alpha_{m}^{(j)}= \pm \sqrt{k_{0}^{2} \varepsilon_{j}-q_{m}^{2}}(j=a, b, s)$ - поперечное волновое число $m$-й пространственной гармоники в $j$-й среде. Используя уравнения (4)-(6) и уравнения Максвелла в каждой среде в фурье-представлении, после очевидных (но достаточно громоздских) алгебраических преобразований получим следующие соотношения между тангенциальными фурье-компонентами электрического поля и поверхностной плотности тока в плоскостях графеновых полосок:

$$
\begin{gathered}
E_{x m}^{(b)}(d)=Z_{m}^{(1,1)} I_{x m}(d)+Z_{m}^{(1,2)} I_{x m}(0)+\delta_{m 0} \gamma^{(1)} E_{x}^{(0)}, \\
E_{x m}^{(b)}(0)=Z_{m}^{(2,1)} I_{x m}(d)+Z_{m}^{(2,2)} I_{x m}(0)+\delta_{m 0} \gamma^{(2)} E_{x}^{(0)}
\end{gathered}
$$

где

$$
\begin{gathered}
Z_{m}^{(1,1)}=-\frac{\alpha_{m}^{(a)} \alpha_{m}^{(b)} \alpha_{m}^{(s)} \varepsilon_{b}}{\omega \varepsilon_{0} \chi_{m}}\left[1+\exp \left(2 i \alpha_{m}^{(b)} d\right)\right] \\
+\frac{\alpha_{m}^{(a)} \alpha_{m}^{(b)} \alpha_{m}^{(s)} \varepsilon_{s}}{\omega \varepsilon_{0} \chi_{m}}\left[1-\exp \left(2 i \alpha_{m}^{(b)} d\right)\right] \\
Z_{m}^{(1,2)}=-\frac{\alpha_{m}^{(a)} \alpha_{m}^{(b)} \alpha_{m}^{(s)} \varepsilon_{b}}{\omega \varepsilon_{0} \chi_{m}} 2 \exp \left(i \alpha_{m}^{(b)} d\right) \\
Z_{m}^{(2,1)}=-\frac{\alpha_{m}^{(a)} \alpha_{m}^{(b)} \alpha_{m}^{(s)} \varepsilon_{b}}{\omega \varepsilon_{0} \chi_{m}} 2 \exp \left(i \alpha_{m}^{(b)} d\right)=Z_{m}^{1,2} \\
Z_{m}^{(2,2)}=-\frac{\alpha_{m}^{(b)} \alpha_{m}^{(b)} \alpha_{m}^{(s)} \varepsilon_{a}}{\omega \varepsilon_{0} \chi_{m}}\left[1-\exp \left(2 i \alpha_{m}^{(b)} d\right)\right] \\
-\frac{\alpha_{m}^{(a)} \alpha_{m}^{(b)} \alpha_{m}^{(s)} \varepsilon_{b}}{\omega \varepsilon_{0} \chi_{m}}\left[1+\exp \left(-2 i \alpha_{m}^{(b)} d\right)\right] \\
\gamma^{(1)}=\frac{\varepsilon_{0} \varepsilon_{a} \omega}{\alpha_{0}^{(a)}} 2 \exp \left(-\alpha_{0}^{(a)} d\right) Z_{0}^{(1,1)} \\
\chi_{m}=\left(\begin{array}{c}
\varepsilon_{0} \varepsilon_{a} \omega \\
\alpha_{0}^{(a)}
\end{array} 2 \exp \left(-\alpha_{0}^{(a)} d\right) Z_{0}^{(2,1)}\right. \\
\left.\gamma_{m}^{(b)} \varepsilon_{a}+\alpha_{m}^{(a)} \varepsilon_{b}\right)\left(\alpha_{m}^{(s)} \varepsilon_{b}-\alpha_{m}^{(b)} \varepsilon_{s}\right) \\
\left.2 i \alpha_{m}^{(b)} d\right)\left(\alpha_{m}^{(b)} \varepsilon_{a}-\alpha_{m}^{(a)} \varepsilon_{b}\right)\left(\alpha_{m}^{(s)} \varepsilon_{b}+\alpha_{m}^{(b)} \varepsilon_{s}\right)
\end{gathered}
$$

Запишем закон Ома $I(x, 0)=\sigma(\omega, x, 0) E(x, y)$ в плоскости графеновых микрополосок $y=0$ и $I(x, d)=\sigma(\omega, x, d) E(x, y) \quad$ при $\quad y=d, \quad$ принимая 
во внимание вид зависимости разрывной функции проводимости от координат

$$
\sigma(\omega, x, 0)=\left\{\begin{array}{lll}
\sigma_{w, 0} & \text { при } & 0<x<w \\
0 & \text { при } & w<x<L
\end{array}\right.
$$

в плоскости $y=0$ и

$$
\sigma(\omega, x, d)=\left\{\begin{array}{lll}
\sigma_{w, d} & \text { при } & 0<x<w, \\
0 & \text { при } & w<x<L
\end{array}\right.
$$

в плоскости $y=d$. В данной постановке задачи $\sigma_{w, 0}=\sigma_{w, d}=\sigma_{\mathrm{Gr}}(\omega)$. Сформулируем систему, состоящую из двух связанных интегральных уравнений относительно поверхностной плотности тока $I_{x}(x, d)$ на графеновой микрополоске в плоскости $y=d$ и относительно поверхностной плотности тока $I_{x}(x, 0)$ на графеновой микрополоске в плоскости $y=0$ :

$$
\begin{aligned}
& I_{x}(x, d)-\sigma_{\mathrm{Gr}}(\omega) \gamma^{(1)} E_{x}^{(0)} \\
& =\int_{0}^{w} I_{x}\left(x^{\prime}, d\right) G_{m}^{(1,1)}\left(x, x^{\prime}\right) d x^{\prime}+\int_{0}^{w} I_{x}\left(x^{\prime}, 0\right) G_{m}^{(1,2)}\left(x, x^{\prime}\right) d x^{\prime},
\end{aligned}
$$

$$
\begin{aligned}
& I_{x}(x, 0)-\sigma_{\mathrm{Gr}}(\omega) \gamma^{(2)} E_{x}^{(0)} \\
& =\int_{0}^{w} I_{x}\left(x^{\prime}, d\right) G_{m}^{(2,1)}\left(x, x^{\prime}\right) d x^{\prime}+\int_{0}^{w} I_{x}\left(x^{\prime}, 0\right) G_{m}^{(2,2)}\left(x, x^{\prime}\right) d x^{\prime},
\end{aligned}
$$

где

$$
\begin{aligned}
& G_{m}^{(1,1)}\left(x, x^{\prime}\right)=\sigma_{\mathrm{Gr}}(\omega) \frac{1}{L} \sum_{m} Z_{m}^{(1,1)} \exp \left[i q_{m}\left(x-x^{\prime}\right)\right], \\
& G_{m}^{(1,2)}\left(x, x^{\prime}\right)=\sigma_{\mathrm{Gr}}(\omega) \frac{1}{L} \sum_{m} Z_{m}^{(1,2)} \exp \left[i q_{m}\left(x-x^{\prime}\right)\right], \\
& G_{m}^{(2,1)}\left(x, x^{\prime}\right)=\sigma_{\mathrm{Gr}}(\omega) \frac{1}{L} \sum_{m} Z_{m}^{(2,1)} \exp \left[i q_{m}\left(x-x^{\prime}\right)\right], \\
& G_{m}^{(2,2)}\left(x, x^{\prime}\right)=\sigma_{\mathrm{Gr}}(\omega) \frac{1}{L} \sum_{m} Z_{m}^{(2,2)} \exp \left[i q_{m}\left(x-x^{\prime}\right)\right]
\end{aligned}
$$

- ядра интегральных уравнений.

Полученная система интегральных уравнений Фредгольма второго рода (9), (10) решалась численно методом Галеркина с разложением двух искомых функций ( $x$-компонент плотности поверхностного электрического тока $I_{x}(x, 0)$ и $\left.E_{x}(x, d)\right)$ в ряд на каждой полоске графена по ортогональным полиномам Лежандра. Индуцированные электрические и магнитные поля в любой точке структуры могут быть найдены с помощью выражений (7) и уравнений Максвелла в каждой из сред.

Длина волны резонансной плазмонной моды, возбуждаемой в графене падающей ТГц волной, определяется периодом $L$ металлической решетки. Поскольку длина волны плазмона в графене значительно (на порядок величины и более) меньше, чем длина электромагнитной волны на той же частоте [4], для возбуждения плазмонных резонансов в структуре необходимо выполнение условия $L \ll 2 \pi / k_{0}$. В этом случае только нулевые фурье-гармоники $(m=0)$ соответствуют бегущим волнам, излучаемым в окружающую среду и в подложку, а все фурье-гармоники более высокого порядка характеризуют эванесцентные поля, убывающие при $y \rightarrow \pm \infty$. Следовательно, коэффициенты отражения $(R)$ и пропускания $(\widetilde{T})$ исследуемой структуры в области дальнего поля могут быть вычислены соответственно как $R=\left|E_{x 0}(d)\right|^{2} /\left|E^{(0)}\right|^{2}$ и $\widetilde{T}=\left|E_{x 0}(0)\right|^{2} \sqrt{\varepsilon_{s}} /\left|E^{(0)}\right|^{2}$. Коэффициент поглощения рассчитывается как $A=P_{\mathrm{abs}} / P_{0}$, где $P_{0}-$ ТГц мощность, падающая на единицу площади структуры,

$$
P_{\mathrm{abs}}=\frac{1}{2 L} \int_{0}^{L} \operatorname{Re}[\sigma(\omega, x)]\left|E_{x}(x, 0)\right|^{2} d x
$$

- усредненная поглощенная ТГц мощность на единице площади структуры. Коэффициент усиления определяется как $K=-A$. Закон сохранения энергии $R+\widetilde{T}+A=1$ выполняется как в режиме поглощения $(A>0)$, так и в режиме усиления $(A<0)$.

Расчеты выполнены для реалистичных параметров исследуемой структуры (рис. 1) с периодом 750 нм при комнатной температуре и $\tau=1$ пс. Период структуры состоит из двух участков: графеновой полоски шириной $w=500$ нм, и просвета шириной 250 нм, диэлектрическая проницаемость барьерного слоя и диэлектрической подложки $\varepsilon_{b}=\varepsilon_{s}=4.0$ (алмазоподобный углерод DLC или $\mathrm{SiCN}$, например). Энергия квазиуровня Ферми оптически накачанного графена полагалась равной 20 мэВ, что соответствует концентрации носителей заряда в

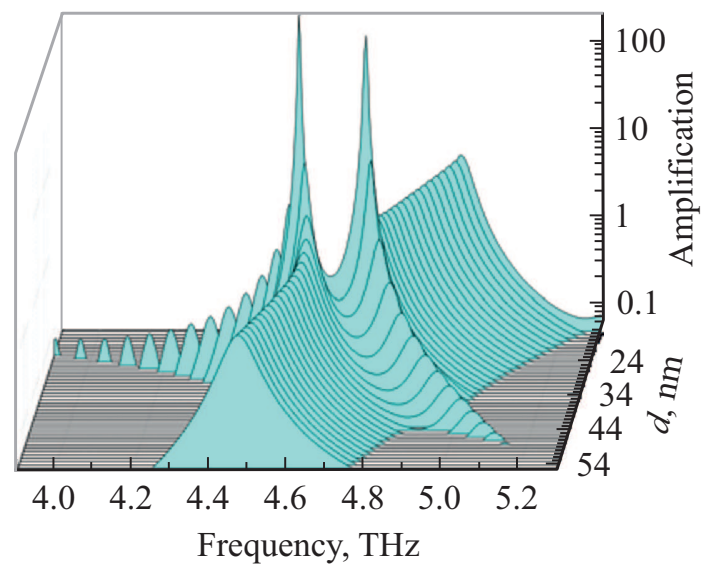

Рис. 3. Коэффициент усиления ТГц мощности в зависимости от частоты падающей ТГц волны и толщины барьерного слоя $d$ для плазменных резонансов. Период графеновой решетки $L=750$ нм, ширина графеновой микрополоски $w=500$ нм. Величина энергии квазиуровня Ферми 20 мэВ. Расчеты приведены для комнатной температуры и $\tau=1$ пс. 


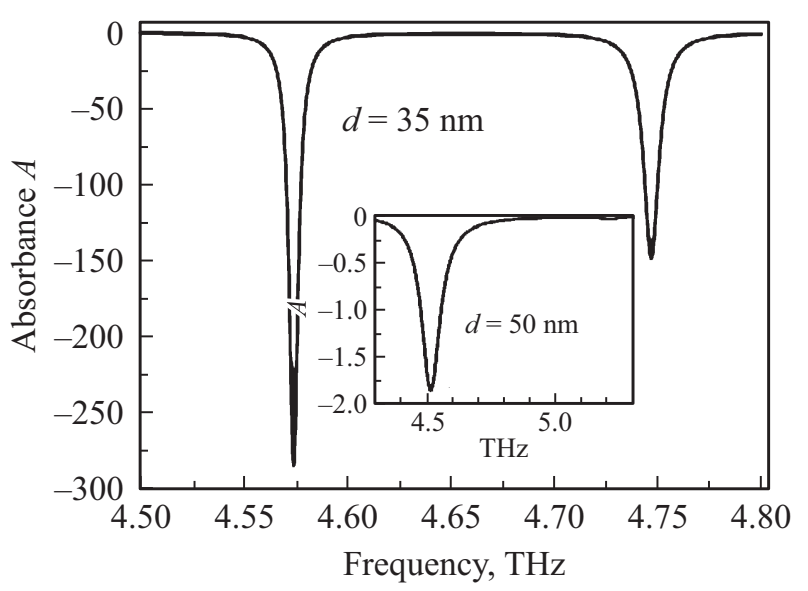

Pис. 4. Коэффициент поглощения ТГц мощности в зависимости от частоты падающей ТГц волны при фиксированной толщине барьерного слоя $d=35$ нм в области антикроссинга двух различных плазменных мод. Период графеновой решетки $L=750$ нм, ширина графеновой микрополоски $w=500$ нм. Величина энергии квазиуровня Ферми 20 мэВ. Расчеты приведены для комнатной температуры и $\tau=1$ пс. На вставке - коэффициент усиления ТГц мощности в зависимости от частоты падающей ТГц волны при фиксированной толщине барьерного слоя $d=500$ нм вдали от области антикроссинга. графене $\sim 2.9 \cdot 10^{10} \mathrm{~cm}^{-2}$, поскольку концентрация носителей заряда в графене связана с энергией Ферми как $N_{n(p)} \propto E_{\mathrm{F}}^{2}\left(\pi \hbar^{2} V_{\mathrm{F}}^{2}\right)[24]$, где $V_{\mathrm{F}}=10^{8} \mathrm{~cm} / \mathrm{c}-$ скорость Ферми.

\section{3. Результаты расчетов и их обсуждение}

Двухслойный периодический планарный массив графеновых микрополосок представляет собой эффективный элемент связи плазмонов с падающим ТГц излучением и одновременно он образует распределенный планарный резонатор для плазмонных мод, возбуждаемых или генерируемых в графене соответственно падающим ТГц излучением или стимулированной плазмонной эмиссией. Такая графеновая двойная периодическая решетка поддерживает плазмонные коллективные моды, колеблющиеся на всей площади структуры [25].

На рис. 3 показан вычисленный спектр усиления ТГц излучения в зависимости от частоты падающей волны и толщины диэлектрического барьерного слоя, разделяющего два периодических массива графеновых микрополосок. Рассматриваемая система поддерживает

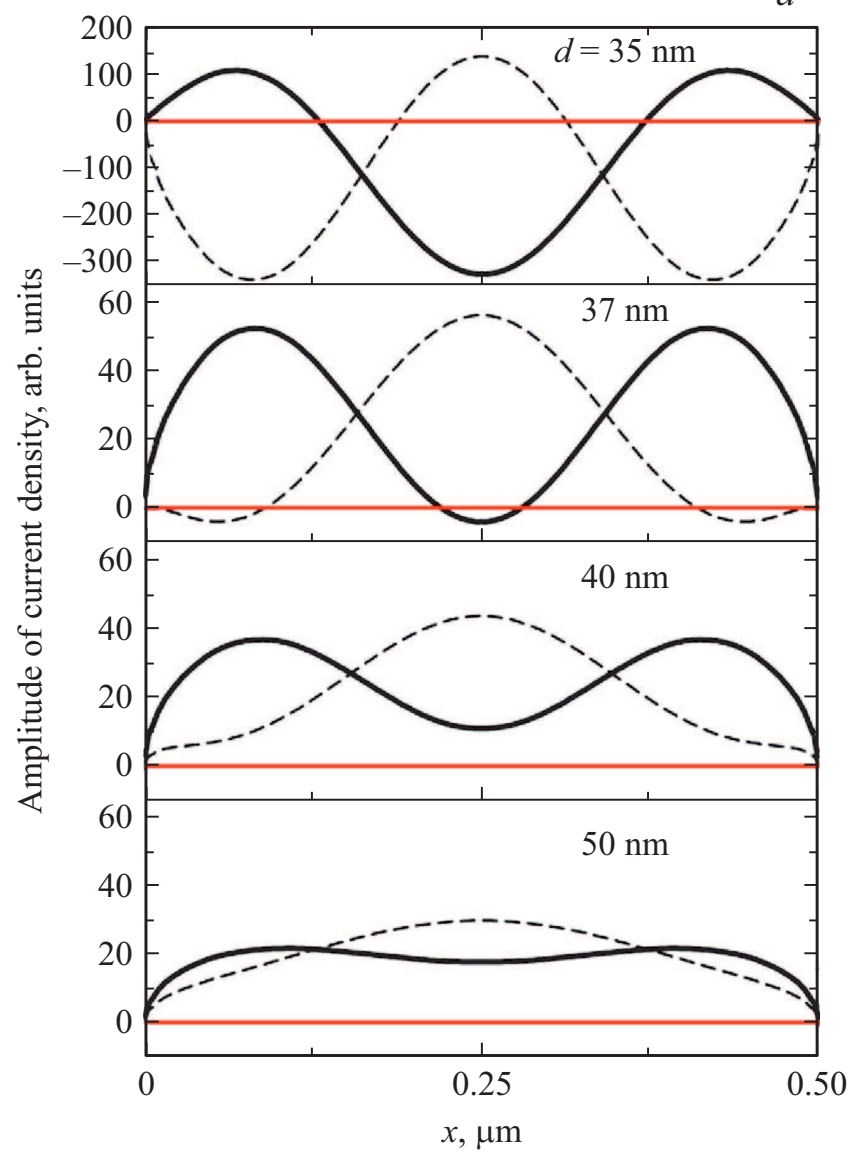

$b$

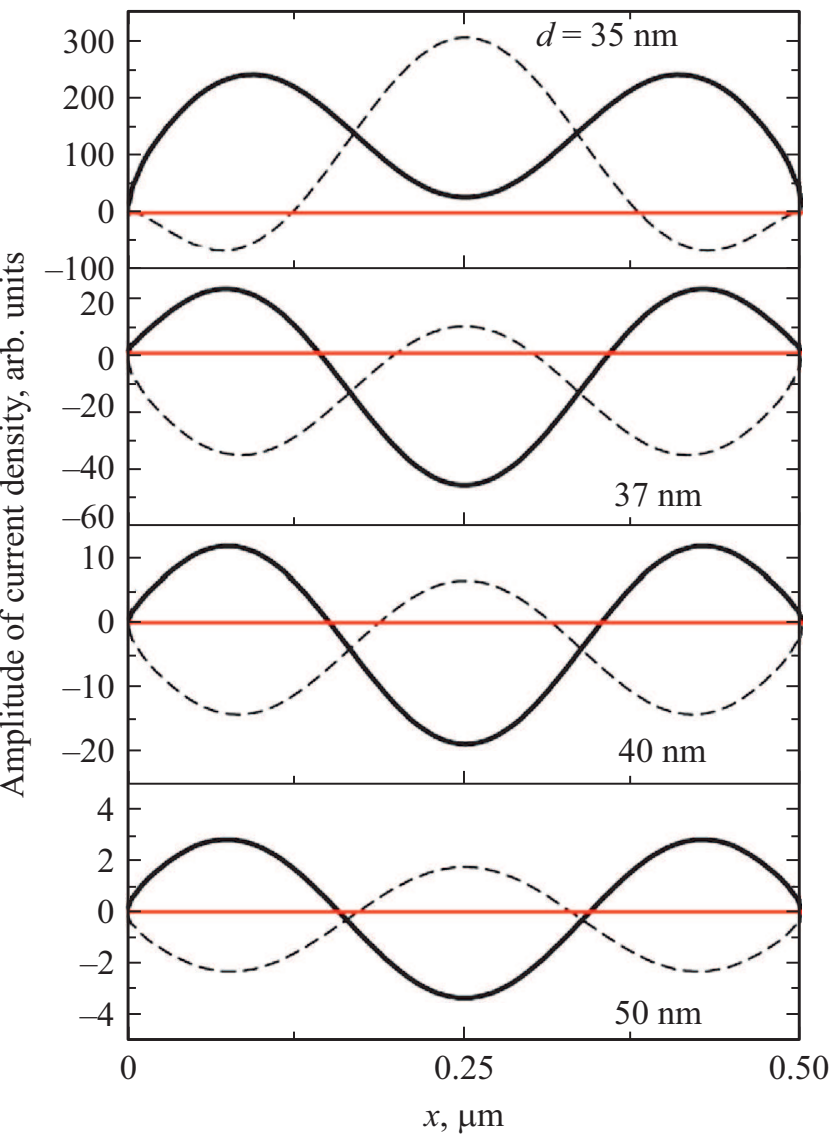

Рис. 5. Распределение амплитуд поверхностной плотности тока фундаментальной оптической плазмонной моды $(a)$ и первой высшей акустической плазмонной моды $(b)$ в графеновой микрополоске в плоскостях $y=d$ (штриховая линия) и $y=0$ (сплошная линия) при различной толщине барьерного слоя между плоскостями. 
оптические и акустические плазмонные моды. На рис. 3 показаны фундаментальная оптическая и первая высшая акустическая моды. Резонансные частоты оптической и акустической мод изменяются противоположным образом с изменением толщины изолирующего диэлектрического слоя $d$, что делает возможным режим их антикроссинга. Максимальное усиление достигается в окрестности режима антикроссинга за счет взаимодействия фундаментальной оптической и первой высшей акустической плазмонных мод. В режиме антикроссинга оптическая плазмонная мода гибридизуется с акустической, в результате чего происходит резкое уменьшение радиационной ширины плазмонного резонанса и увеличение добротности резонанса для каждой из двух взаимодействующих мод. При этом достигаются большие коэффициенты усиления $(\sim 300)$ на двух различных резонансных частотах плазмонных мод (рис. 4). Отметим, что с уменьшением толщины изолирующего диэлектрического слоя $d$ с 50 до 35 нм вычисленная добротность резонанса оптической плазмонной моды (рис. 4) резко возрастает с 45 до 850. Распределение поверхностной плотности тока в графеновых микрополосках в верхней и нижней плоскостях, при различных толщинах барьерного слоя $d$ между плоскостями, показанное на рис. $5, a, b$, иллюстрирует гибридный характер двух различных плазмонных мод. Внизу на рис. 5, $a$ и $b$ показаны соответственно симметричное распределение поверхностной плотности тока фундаментальной оптической плазмонной моды и антисимметричное распределение первой высшей акустической плазмонных мод при $d=50$ нм. С уменьшением параметра $d$ плазмонные моды непрерывно трансформируются и при $d=35$ нм (см. рис. 5, $a, b$, вверху) гибридизуются.

\section{4. Заключение}

Показано, что двухслойный периодический планарный массив графеновых микрополосок представляет собой эффективный элемент связи плазмонов с падающим ТГц излучением и поддерживает оптические и акустические плазмонные моды.

Предложен метод контроля радиационной ширины плазмонного резонанса (и как следствие добротности резонанса) для каждой из двух взаимодействующих мод путем изменения толщины диэлектрического барьерного слоя между двумя слоями массивов графеновых микрополосок. Показано, что наибольшее усиление ТГц излучения в двухслойном периодическом массиве графеновых микрополосок достигается на двух различных резонансных частотах плазмонных мод вблизи режима антикроссинга даже при малой энергии квазиуровня Ферми при комнатной температуре. Полученные результаты показывают возможность создания компактных, частотно перестраиваемых наноразмерных плазмонных усилителей и генераторов в ТГц частотном диапазоне на основе инвертированного графена.

\section{Финансирование работы}

Работа выполнена в рамках государственного задания и частично поддержана Российским фондом фундаментальных исследований (проект РФФИ № 18-37-20004).

\section{Конфликт интересов}

Авторы заявляют, что у них нет конфликта интересов.

\section{Список литературы}

[1] R.R. Nair, P. Blake, A.N. Grigorenko, K.S. Novoselov, T.J. Booth, T. Stauber, N.M. Peres, A.K. Geim. Science, 320, 1308 (2008).

[2] K.S. Novoselov, A.K. Geim, S.V. Morozov, D. Jiang, M.I. Katsnelson, I.V. Grigorieva, S.V. Dubonos, A.A. Firsov. Nature, 438, 197 (2005).

[3] Y. Zhang, Y.-W. Tan, H.L. Stormer, P. Kim. Nature, 438, 201 (2005).

[4] J. Chen, M. Badioli, P. Alonso-González, S. Thongrattanasiri, F. Huth, R. Hillenbrand, F.H.L. Koppens. Nature, 487, 77 (2012).

[5] Z. Fei, A.S. Rodin, G.O. Andreev, W. Bao, A.S. McLeod, M. Wagner, L.M. Zhang, Z. Zhao, M. Thiemens, G. Dominguez, M.M. Fogler, A.H. Castro Neto, C.N. Lau, F. Keilmann, D.N. Basov. Nature, 487, 82 (2012).

[6] V. Ryzhii, M. Ryzhii, T. Otsuji. J. Appl. Phys., 101, 083114 (2007).

[7] A. Satou, F.T. Vasko, V. Ryzhii. Phys. Rev. B, 7, 1158431 (2008).

[8] A.A. Dubinov, V.Y. Aleshkin, M. Ryzhii, T. Otsuji, V. Ryzhii. Appl. Phys. Exp., 2, 092301 (2009).

[9] A. Satou, T. Otsuji, V. Ryzhii. Jpn. J. Appl. Phys., 50, 070116 (2011).

[10] A. Satou, V. Ryzhii, Y. Kurita, T. Otsuji. J. Appl. Phys., 113, 143108 (2013).

[11] F. Rana. IEEE Trans. Nanotechn., 7, 91 (2008).

[12] S. Boubanga-Tombet, S. Chan, T. Watanabe, A. Satou, V. Ryzhii, T. Otsuji. Phys. Rev. B, 85, 035443 (2012).

[13] T. Winzer, E. Malic, A. Knorr. Phys. Rev. B, 87, 165413 (2013).

[14] R. Jago, T. Winzer, E. Malic. Phys. Status Solidi B, 252 (11), 2456 (2015).

[15] G. Alymov, V. Vyurkov, V. Ryzhii, A. Satou, D. Svintsov. Phys. Rev. B, 97, 205411 (2018).

[16] T. Li, L. Luo, M. Hupalo, J. Zhang, M.C. Tringides, J. Schmalian, J. Wang. Phys. Rev. Lett., 108, 167401 (2012).

[17] I. Gierz, M. Mitrano, J.C. Petersen, C. Cacho, I.C.E. Turcu, E. Springate, A. Stöhr, A. Köhler, U. Starke, A. Cavalleri. J. Phys.: Condens. Matter, 27, 164204 (2015).

[18] M.T. Mihnev, F. Kadi, C.J. Divin, T. Winzer, S. Lee, C.-H. Liu, Z. Zhong, C. Berger, W.A. de Heer, E. Malic, A. Knorr, T.B. Norris. Nature Commun., 7, 11617 (2016).

[19] I. Gierz. J. Electron. Spectr. Rel. Phenomena, 219, 53 (2017).

[20] J.H. Strait, H. Wang, S. Shivaraman, V. Shields, M. Spencer, F. Rana. Nano Lett., 11, 4902 (2011).

[21] A. Dubinov, V.Y. Aleshkin, V. Mitin, T. Otsuji, V. Ryzhii. J. Phys.: Condens. Matter, 23, 145302 (2011).

[22] C.H. Gan, H.S. Chu, E.P. Li. Phys. Rev. B, 85, 125431 (2012). 
[23] L.A. Falkovsky, A.A. Varlamov. Eur. Phys. J. B, 56, 281 (2007).

[24] A.H. Castro Neto, F. Guinea, N.M.R. Peres, K.S. Novoselov, A.K. Geim. Rev. Mod. Phys., 81, 109 (2009).

[25] V.V. Popov, O.V. Polischuk, A.R. Davoyan, V. Ryzhii, T. Otsuji, M.S. Shur. Phys. Rev. B, 86, 195437 (2012).

Редактор Л.В. Шаронова

\title{
Amplification of terahertz radiation by high-quality resonant plasmons in graphene periodic double-layer structure in the anticrossing regime of plasmon modes
}

O.V. Polischuk ${ }^{1}$, D.V. Fateev ${ }^{1,2}$, V.V. Popov ${ }^{1}$

${ }^{1}$ Kotelnikov Institute of

Radio Engineering and Electronics,

Russian Academy of Sciences,

410019 Saratov, Russia

${ }^{2}$ National Research Saratov State University, 410012 Saratov, Russia

\begin{abstract}
The amplification spectrum of terahertz radiation in a double-layer active graphene nanostructure, which is two identical periodic arrays of graphene microribbons separated by a thin dielectric barrier layer, is theoretically investigated. The system in question supports optical and acoustic plasmon modes. The resonance frequencies of the optical and acoustic modes change in the opposite way with a change in the thickness of the insulating dielectric barrier layer, which makes the anticrossing regime of plasmon modes possible. It is shown that the investigated graphene structure exhibits a strong plasmon response and a giant amplification of terahertz radiation at the two plasma resonance frequencies in the vicinity of the anticrossing regime between optical and acoustic plasmon modes at room temperature.
\end{abstract}

